\title{
Implementation of Microservice-based Network Traffic Logger
}

\author{
Chakrit Phain and Yachai Limpiyakorn ${ }^{+}$ \\ Department of Computer Engineering, Chulalongkorn University, Bangkok 10330, Thailand
}

\begin{abstract}
For the benefits of information security, data storage management is often performed under supervision of official regulations, such as ISO/IEC 27001. One of the topics addressing operations security specifies the requirements of logging and monitoring to record events and generate evidence. As the vast amount of traffic flowing over the internet continues to grow, this paper presents the implementation of network traffic logger using Microservices to promote scalability. It also enables isolated upgrades and new features without necessarily disrupting other services and the teams developing other services. With the assistance of the microservice architecture, the log management system would be more easily managed and scaled.
\end{abstract}

Keywords: traffic log management, information security, microservices.

\section{Introduction}

ISO/IEC 27001 [1] is an information security standard, part of the ISO/IEC 27000 family of standards. Following this standard will help organizations keep information assets secure. The standard specifies a management system that is intended to bring information security under management control. To achieve operations security, the topic A.12.4 specifies the requirements of Logging and Monitoring in order to record events and generate evidence. The storage of network traffic logs consists of several parts, such as Radius $\log$ and Firewall log. With centralized log management, a syslog protocol used to send system logs or event messages to a specific server aims to gather all different log messages in one location so as to be easy to form into an aggregate and query. Hence, logging network traffic in a monolithic format will cause dependent of each other, and when they are retrieved, optimum capacity is reached without necessary. As the vast amount of traffic flowing over the internet continues to grow, if the data can be separated and classified based on the purpose of use, then the burden of data retrieval can be reduced in a more efficient manner.

Over the past few years, many large organizations evolve their technology stack to Microservices, also known as microservice architecture. The architectural style structures an application as a collection of loosely coupled services to implement business capabilities. That is, componentization via services is organized around business capability. The operation is prone to isolate each service in its own. Microservices also changes the scalability model of an application. Each service can be individually scaled, with the result being that the application is scaled more efficiently. The scaling in Microservices can be divided into 3 types: 1) $\mathrm{X}$-axis, 2) $\mathrm{Y}$-axis, and 3) Z-axis [2]. $\mathrm{X}$-axis is a load balancing scaling, sometimes referred to as scale-out or horizontal scale. It is built by cloning to have the same features. The Y-axis scaling is generally in the form of service classification, such as "login", "checkout", "search". Finally, the Z-axis scaling is similar to that of X-axis which relies on cloning of an instance of the application. The difference is the Z-axis scaling uses a sharding method, i.e. the expanded data would be divided partly by its function as per usage, such as Jan-Mar, Apr-Jun, Jul-Sep, and Oct-Dec.

This paper thus presents an approach to implementing a log management system with microservice design to improve scalability and data retrieval efficiency.

\footnotetext{
+ Corresponding author. Tel.: +668 2218 6959; fax: +668 22186955.
}

E-mail address: Yachai.L@chula.ac.th. 


\section{Microservices}

Microservices is a software architecture that models complex software applications as a suite of independently deployable, small, and modular services. Each service runs a unique process and communicates through a well-defined, lightweight mechanism (REST APIs) [3]. Example as shown in Fig. 1, a single application as a suite of small services runs in its own process and communicating with lightweight mechanisms, often HTTP Resource API. Each service can be written in any languages, not necessary to be the same language. Regarding databases, it is not required to share the same database as one service owns some pieces of data, and can create Build, Test, and Release function without having any effect with existing functions. Benefits of microservice architectural approach include [5] :

- Tackle the problem of complexity

- Enable each service to be developed independently by a team that is focused on that service

- Enable each microservice to be deployed independently

- Enable each service to be scaled independently

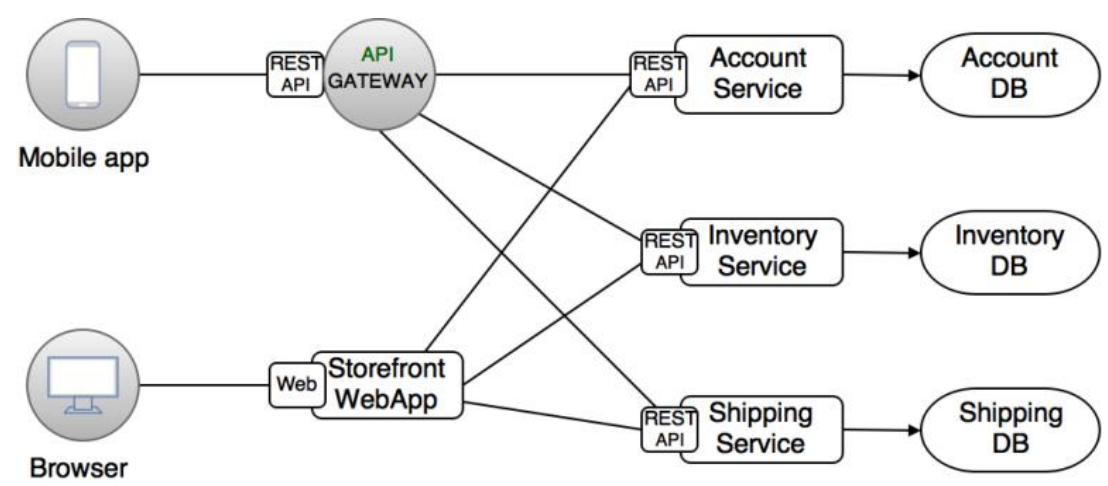

Fig. 1: Example application modelled with microservice architecture [4].

\section{Implementation}

In this work, the implementation of traffic logger consists of two main service components: Radius log service and Firewall log service, each of which connects to its own API and database as shown in Fig. 2. Table 1 describes the information stored in Radius logs including Username, Source IP (Framed-IP-Address), Status (Acct-Status-Type), and Timestamp. Table 2 describes the attributes contained in Firewall logs including Source IP, Source Port, Destination IP, Destination Port, and Timestamp.

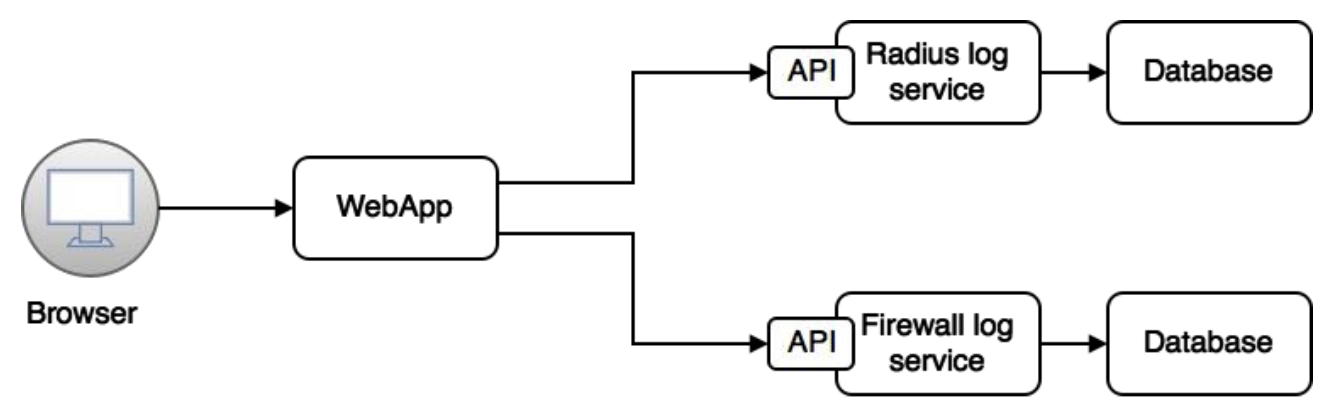

Fig. 2: Log data retrieval with Microservices.

Fig. 3 illustrates the traffic logger implemented with Microservices scale cube. The X-axis scale ensures a balanced routing to distribute service workloads in Round-Robin. Scaling on the Y-axis is classified by service types, i.e. services will be divided into 2 parts: Radius and Firewall. The Radius service focuses on the store and retrieval of basic information about authentication, while the Firewall emphasizes on providing the retrieval service for IP at any starting to ending destination and any protocol numbers. Typically, an $\mathrm{x}$ axis splits for each of $\mathrm{z}$-axis split. When scaling on the Z-axis, service workloads are divided based on determined regulations. For example, in Thailand, there is a regulation demanding for maintaining the last 3month of traffic logs. As a result, the X-axis split on Radius services will be further split into months for scaling on the Z-axis. 
Table 1: Radius log description.

\begin{tabular}{|c|c|}
\hline Attribute & Description \\
\hline Timestamp & Record the time that this event occurred \\
\hline User-Name & Name of the user to be authenticated \\
\hline Framed-IP-Address & Contain the IP address of the user \\
\hline Acct-Status-Type & $\begin{array}{l}\text { Indicate whether this Accounting-Request marks the beginning of the user service } \\
\text { (Start) or the end (Stop) }\end{array}$ \\
\hline
\end{tabular}

Table 2: Firewall log description.

\begin{tabular}{l|l}
\hline Attribute & Description \\
\hline Timestamp & Record the time that this event occurred \\
Source IP & Contain the IP address of the user \\
Source Port & Contain the Port of the user used \\
Destination IP & IP address of user access \\
Destination Port & A number used to identify a network service \\
\hline
\end{tabular}

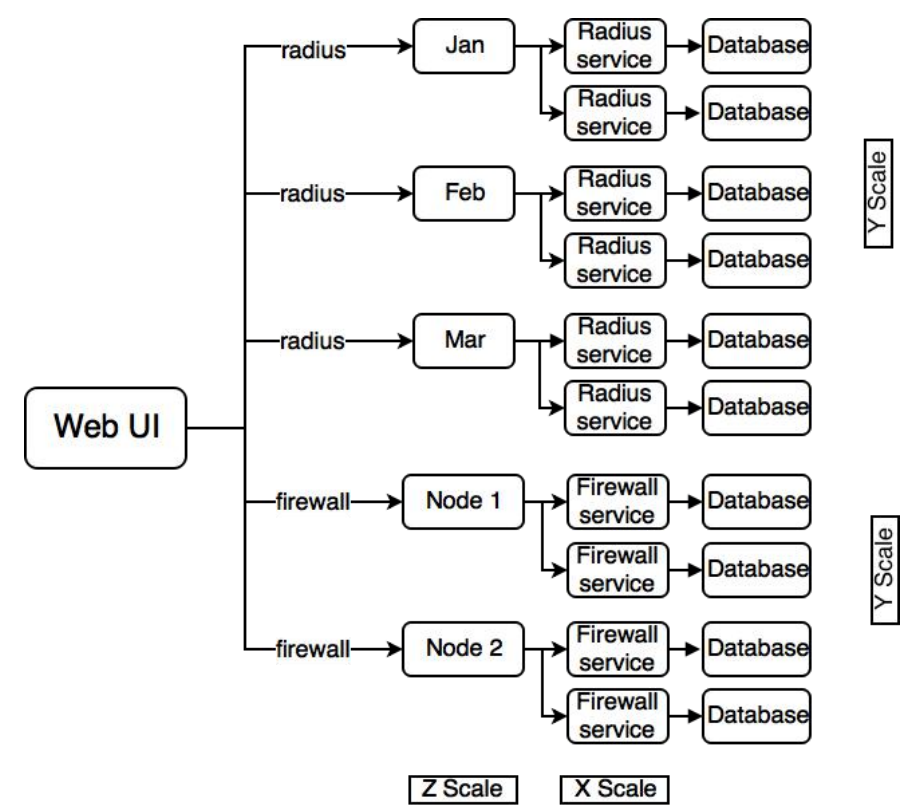

Fig. 3: Traffic logger modelled with Microservices scale cube.

Fig. 4 depicts the procedure to manage data consistency among databases. If a failure occurs at any service, an event will be created and sent to notify all associated services for job cancellation.

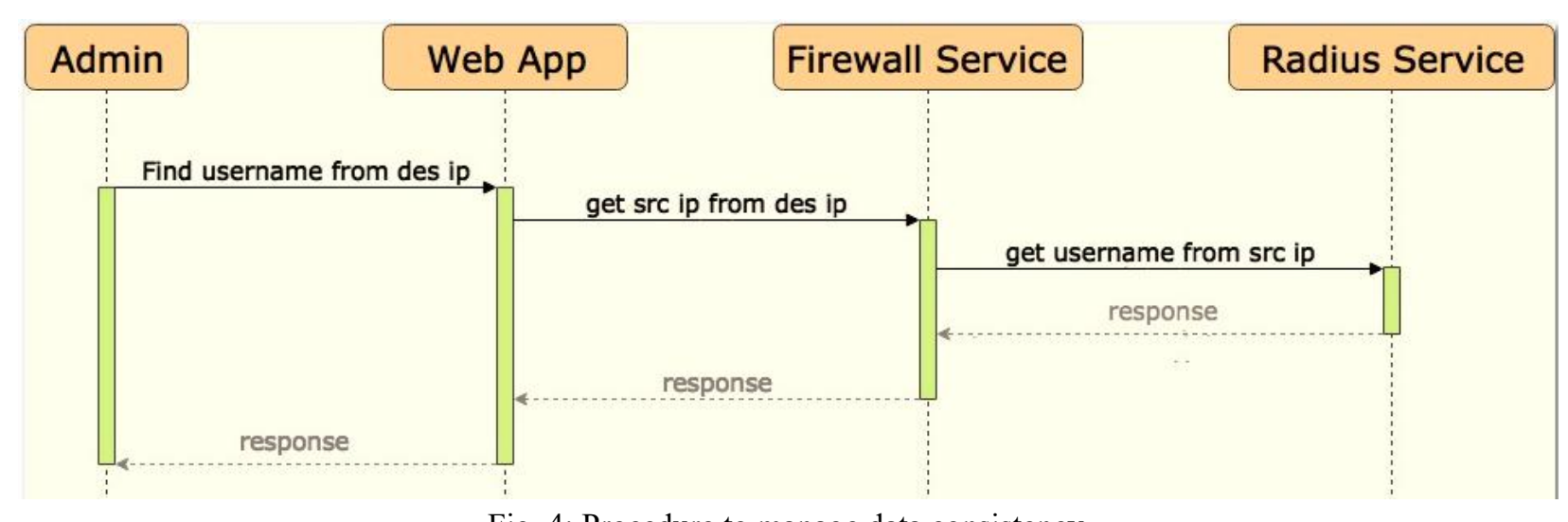

Fig. 4: Procedure to manage data consistency. 
The UI design consists of three main pages: 1) Radius log screen, 2) Firewall log screen, and 3) Mergedlogs screen. Fig. 5 illustrates the UI screen as a result of querying the Radius log service for the retrieval of authentication information occurring within the specified period and the certain IP address. Similarly, Fig. 6 illustrates the UI screen as a result of querying the Firewall log service for the retrieval of network traffic information occurring within the specified search keywords.

\section{LogQuery Radlus $\log$ Frrewall log Merge $\log$}

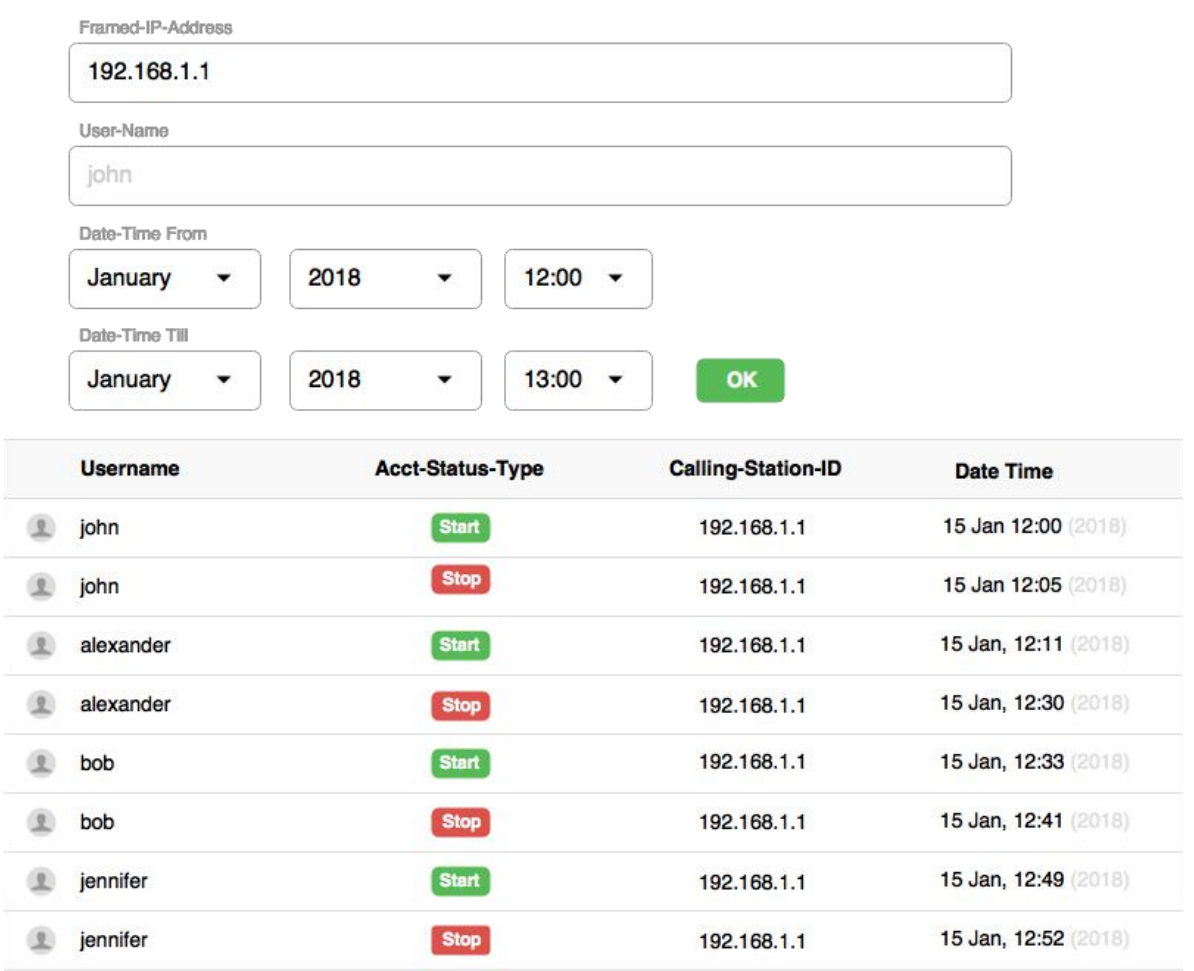

Fig. 5: UI of the result of querying Radius log service.

\section{LogQuery Radlus log Frewall log Merge log}

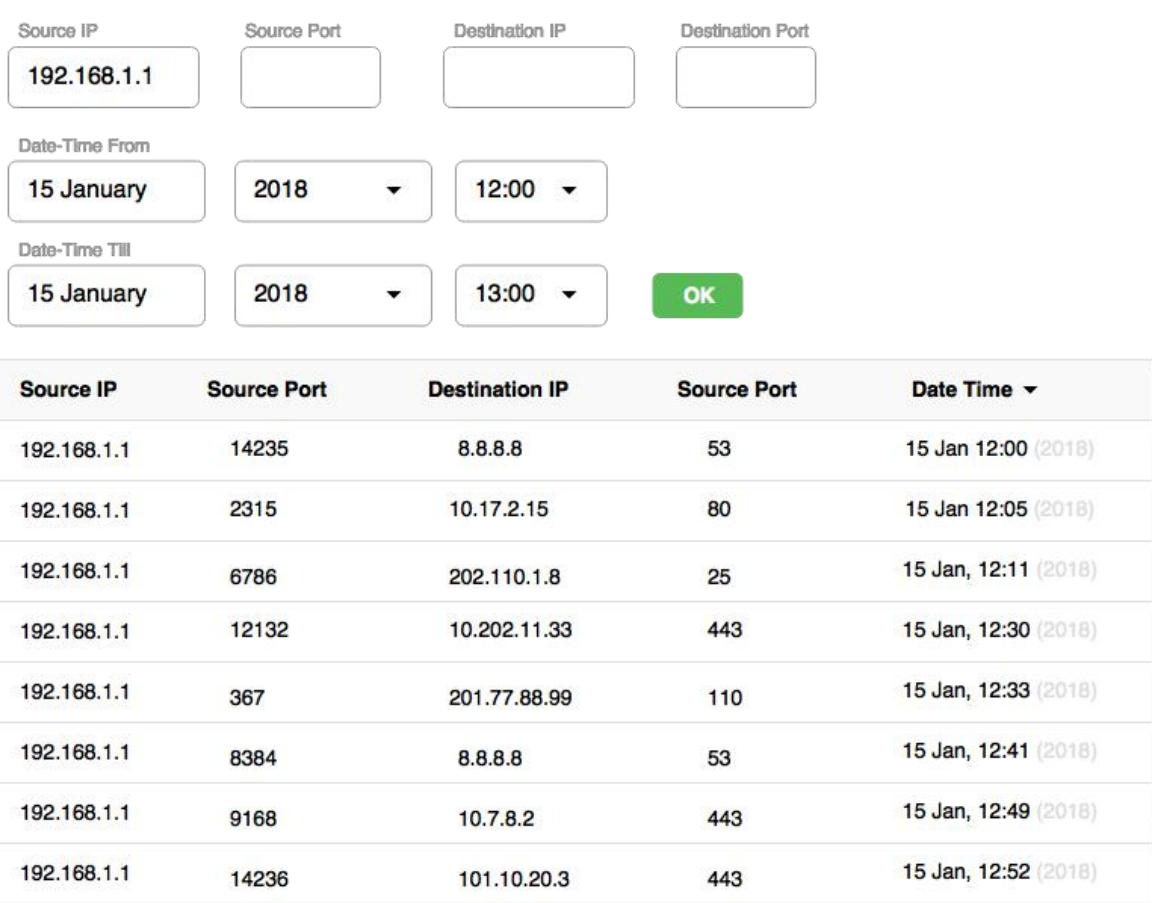

Fig. 6: UI of the result of querying Firewall log service. 
LogQuery Radlus log Firewall log Merge log

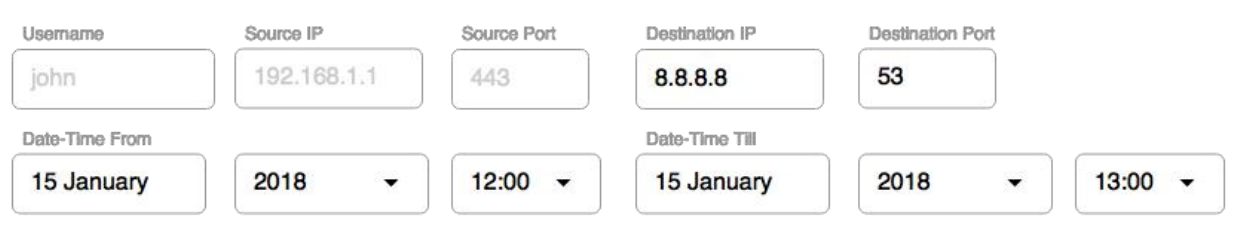

OK

\begin{tabular}{ccccccc}
\hline Username & Source IP & Source Port & Destination IP & Source Port & Date Time - \\
\hline john & 192.168 .1 .1 & 14235 & 8.8 .8 .8 & 53 & 15 Jan 12:00 (2018) \\
\hline john & 192.168 .1 .1 & 2315 & 8.8 .8 .8 & 53 & 15 Jan 12:05 (2018) \\
\hline alexander & 192.168 .1 .3 & 6786 & 8.8 .8 .8 & 53 & 15 Jan, 12:11 (2018) \\
\hline alexander & 192.168 .1 .3 & 12132 & 8.8 .8 .8 & 53 & 15 Jan, 12:30 (2018) \\
\hline bob & 192.168 .1 .12 & 367 & 8.8 .8 .8 & 53 & 15 Jan, 12:33 $(2018)$ \\
\hline bob & 192.168 .1 .12 & 8384 & 8.8 .8 .8 & 53 & 15 Jan, 12:41 $(2018)$ \\
\hline jeniffer & 192.168 .1 .18 & 9168 & 8.8 .8 .8 & 53 & 15 Jan, 12:49 (2018) \\
\hline jeniffer & 192.168 .1 .18 & 14236 & 8.8 .8 .8 & 53 & 15 Jan, 12:52 \\
\hline
\end{tabular}

Fig. 7: Merged- logs screen

Fig. 7 shows the Merged-log page created by joining the information from Radius logs and Firewall logs. The results inform who access to the specified destination IP, the specified destination port, within the specified period of time.

\section{Conclusion}

Scaling in the current centralized log management with monolithic architecture is inefficient, due to the rapid growing number of traffic logs. With the assistance of microservice architecture, the log management system would be more easily managed and scaled. It is found that splitting the Radius log service apart from the Firewall log service would reduce the complexity of design and implementation. Moreover, separating the database connecting to each service would promote the independent among services. As services are modelled as isolated units, it simplifies adding other services in the future without affecting the existing services. Each service can use the type of database that is best suited to its needs and service scaling can be easily operated. Future direction would be investigating the system performance compared to that of the system implemented with a monolithic format.

\section{References}

[1] International Organization for Standardization/ International Electro technical Commission, ISO 27001:2013: Information technology - Security techniques - Information security management systems - Requirements, 2013.

[2] M. L. Abbott and M. T. Fisher : The Art of Scalability: Scalable Web Architecture, Processes, and Organizations for the Modern Enterprise: Addison-Wesley, 2015.

[3] J. Lewis and M. Fowler "Decentralized Data Management" https://martinfowler.com/articles/microservices.html [Accessed: Feb 2018]

[4] C. Richardson, "Pattern: Microservice Architecture" http://microservices.io/patterns/microservices.html [Accessed: Nov 2017]

[5] Microservices from Design to Deployment. NGINX. 2016. pp. 8. 